\title{
Increased intestinal ethanol following consumption of fructooligosaccharides in rats
}

\author{
MASAHIRO YAMAGUCHI ${ }^{1}$, YONGSHOU YANG ${ }^{1}$, MISAKI ANDO ${ }^{1}$, \\ THANUTCHAPORN KUMRUNGSEE ${ }^{1}$, NORIHISA KATO ${ }^{1}$ and YUKAKO OKAZAKI $^{2}$ \\ ${ }^{1}$ Department of Biofunctional Science and Technology, Graduate School of Biosphere \\ Science, Hiroshima University, Higashi-Hiroshima, Hiroshima 739-8528; ${ }^{2}$ Faculty of Human \\ Life Sciences, Fuji Women's University, Ishikari, Hokkaido 061-3204, Japan
}

Received January 4, 2018; Accepted September 10, 2018

DOI: $10.3892 /$ br.2018.1150

\begin{abstract}
Previous studies have suggested that ethanol is a fermentation product of microflora. However, it is unknown whether this ethanol production is elevated by intake of prebiotics. Prebiotics are considered to enhance the production of short-chain fatty acids (SCFAs) as a fermentation product of beneficial bacteria. In the present study, the effect of fructooligosaccharides (FOS) consumption on intestinal ethanol levels was investigated. Rats were fed a diet with or without $10 \%$ FOS for two weeks. Consequently, FOS intake significantly increased ethanol levels per gram of ileum and cecum digesta of the rats (3.5-fold and 1.9-fold, respectively, $\mathrm{P}<0.01$ ). The numbers of cecum Bifidobacterium (producer of ethanol and lactate) were significantly increased by FOS intake $(\mathrm{P}<0.05)$ and correlated with the cecum ethanol levels per gram of cecum $(r=0.626, \mathrm{P}<0.05)$. FOS intake also led to a significant increase in the cecum levels of SCFAs, namely lactate, propionate and $\mathrm{n}$-butyrate $(\mathrm{P}<0.05)$. Furthermore, ethanol levels were significantly correlated with lactate levels $(\mathrm{r}=0.691, \mathrm{P}<0.01)$, but not with propionate or $\mathrm{n}$-butyrate levels $(r=0.449$ and 0.493 , respectively, $P>0.05)$. The current study, to the best of our knowledge, is the first to indicate that FOS intake significantly increases the level of intestinal ethanol. Therefore, dietary FOS may affect the intestinal health status of animals by elevating their ethanol levels, without direct ethanol consumption.
\end{abstract}

Correspondence to: Professor Norihisa Kato, Department of Biofunctional Science and Technology, Graduate School of Biosphere Science, Hiroshima University, 1-4-4 Kagamiyama, Higashi-Hiroshima, Hiroshima 739-8528, Japan

E-mail: nkato@hiroshima-u.ac.jp

Abbreviations: FOS, fructooligosaccharides; SCFAs, short-chain fatty acids; HF, high-fat; qPCR, quantitative polymerase chain reaction; HPLC, high-performance liquid chromatography

Key words: fructooligosaccharides, prebiotics, ethanol, Bifidobacterium, intestine, short-chain fatty acids, rat

\section{Introduction}

Previous studies have suggested that ethanol is a fermentation product of intestinal microflora (1-3). Additionally, it has been reported that ethanol is produced from glucose or fructooligosaccharides (FOS) by several intestinal bacteria, including Bifidobacterium, Lactobacillus fermentum and Weissella confusa (4). However, the extent to which these bacteria are responsible for ethanol production is unknown. Furthermore, there is a lack of studies on the effects of dietary factors, such as prebiotics, on intestinal ethanol production in animals without ethanol consumption. Consumption of prebiotics including FOS and inulin has been reported to enhance the intestinal fermentation process by elevating levels of probiotics including Bifidobacterium and Lactobacillus (5). It has been reported that addition of FOS to culture medium stimulates in vitro growth of Bifidobacterium (6,7). Dietary FOS is resistant to digestion and is metabolised by the microflora in the large intestine into short-chain fatty acids (SCFAs). Increasing data suggest that prebiotics may prevent several chronic diseases, including colon cancer, inflammatory bowel disease, alcoholic steatohepatitis and diabetes, by elevating probiotic and SCFA levels $(8,9)$.

A high intake of ethanol is harmful due to increased risk of several diseases including coronary heart disease, brain diseases and colon cancer (10). Conversely, it has been suggested that low ethanol intake is associated with lower risk of such diseases $(10,11)$. Therefore, it is of interest to elucidate the modulation of intestinal ethanol production by dietary factors. Considering the previous findings, the present study hypothesised that FOS intake may elevate ethanol production by the intestinal microflora. Indeed, the beneficial effects of dietary prebiotics on obese phenotype, impaired gut permeability, cardiac function and hyperlipidemia are particularly prominent in animals on a high-fat (HF) diet $(12,13)$. Therefore, in the current study, the effect of FOS intake on intestinal ethanol levels in rats fed on a HF diet was investigated.

\section{Materials and methods}

Animals and diets. A total of 16 male, specific pathogen-free Sprague-Dawley rats (3 weeks old) were purchased from 
Hiroshima Laboratory Animal Centre (Hiroshima, Japan) and maintained according to the Guide for the Care and Use of Laboratory Animals of Hiroshima University (Hiroshima, Japan). The study was approved by the Research Ethics Committee of Hiroshima University (approval no.: C15-12). The rats were individually housed in an air-conditioned room at $23-24^{\circ} \mathrm{C}$ under a 12 -h light/dark cycle (lights on from 08:00 a.m. to 8:00 p.m. Following acclimatization with a non-purified commercial rodent diet (MF diet for rat and mouse; Oriental Yeast Co., Ltd., Tokyo, Japan) for 3 days, the rats (mean body weight, $68.3 \pm 0.5 \mathrm{~g}$ ) were randomly assigned to one of two groups ( $\mathrm{n}=8$ rats per group) based on experimental diet. The compositions of the experimental HF diets with and without FOS supplementation are summarised in Table I. In these diets, the $30 \%$ beef tallow and $10 \%$ FOS were in accordance with previous studies $(14,15)$. The rats were randomly assigned to one of the two diets supplemented with or without 10\% FOS (w/w; Wako Pure Chemical Industries, Ltd., Osaka, Japan). Equal quantities of the experimental diets were incorporated daily into food cups at 17:00 $(9,10,12,14$ and $15 \mathrm{~g}$ on days 1, 2-4, 5-7, 8-12 and 13-14, respectively) to prevent differences in food intake. Food intake was determined from the food consumed each day until the next day's food was served. The weight of spilled food was recorded daily and appropriately incorporated into calculations of food intake. At the end of the feeding period, the rats were sacrificed by decapitation following brief exposure to 3-4\% isoflurane gas (Wako Pure Chemical Industries, Ltd., Osaka, Japan) mixed with air in an anesthesia chamber. A total of $8 \mathrm{ml}$ blood was collected from the neck, and serum was separated by centrifugation at $2,000 \mathrm{x} \mathrm{g}$ for $20 \mathrm{~min}$ at $4^{\circ} \mathrm{C}$ and stored at $-80^{\circ} \mathrm{C}$. The intestinal digesta was immediately isolated, weighed and stored at $-80^{\circ} \mathrm{C}$ until the subsequent analyses of ethanol, organic acid and beneficial bacteria.

Cecum and serum ethanol. To determine ethanol levels by gas chromatography, $100 \mathrm{mg}$ of the intestinal digesta and $100 \mu \mathrm{l}$ serum were transferred into individual ice-cold $0.6 \mathrm{~N}$ perchloric acid solutions (PCA). The cecum PCA solution was homogenized on ice (Polytron RT-MR2100; Kinematica AG, Littau-Luzern, Switzerland), and the serum PCA solution was mixed for $10 \mathrm{sec}$ on a vortex-mixer (17). The extracted samples were centrifuged at $10,000 \times \mathrm{g}$ at $4^{\circ} \mathrm{C}$ for $5 \mathrm{~min}$, and the supernatant was stored as $1.0 \mathrm{ml}$ samples collected in $20 \mathrm{ml}$ glass vials at $-80^{\circ} \mathrm{C}$ until measurement. 1-Propanol at a final concentration of $100 \mathrm{ppm}$ was included as internal standard prior to analysis. A gas-chromatograph (GC-2014; Shimadzu Corporation, Kyoto, Japan) equipped with a flame ionization detector was used for analysis. A headspace autosampler (HT2000H; ALPHA M.O.S. Japan K.K., Tokyo, Japan) was used to load the analyses. DB-WAX capillary columns $(1.0 \mu \mathrm{m}$ thickness, $30 \mathrm{~m}$ length, $0.53 \mathrm{~mm}$ internal diameter) from Agilent Technologies Japan, Ltd. (Tokyo, Japan) were applied. Samples were incubated for $30 \mathrm{~min}$ at $65^{\circ} \mathrm{C}$ in the head-space autosampler. Samples $(0.5 \mathrm{ml})$ were injected into the column at a rate of $1 \mathrm{ml} / \mathrm{min}$ with the injector maintained at $200^{\circ} \mathrm{C}$ and detector at $250^{\circ} \mathrm{C}$. An initial temperature of $40^{\circ} \mathrm{C}$ was maintained for $1 \mathrm{~min}$ and then the column oven temperature was increased from 40 to $80^{\circ} \mathrm{C}$ at a rate of $8^{\circ} \mathrm{C} / \mathrm{min}$ and then maintained at $80^{\circ} \mathrm{C}$ for $1 \mathrm{~min}$. The column oven temperature
Table I. Composition of the experimental diets.

\begin{tabular}{lcc}
\hline & \multicolumn{2}{c}{ Proportion of diet (\%) } \\
\cline { 2 - 3 } Material & Control & $10 \%$ FOS \\
\hline Beef tallow $^{\mathrm{a}}$ & 30.0 & 30.0 \\
Casein $^{\mathrm{b}}$ & 20.0 & 20.0 \\
L-Cystine $^{\mathrm{a}}$ & 0.3 & 0.3 \\
Vitamin mixture $^{\mathrm{c}}$ & 1.0 & 1.0 \\
Mineral mixture $^{\mathrm{c}}$ & 3.5 & 3.5 \\
Cellulose $^{\mathrm{c}}$ & 5.0 & 5.0 \\
Sucrose $^{\mathrm{a}}$ & 20.0 & 20.0 \\
Corn starch $^{\mathrm{c}}$ & 20.2 & 10.2 \\
FOS $^{\mathrm{d}}$ & - & 10.0 \\
\hline
\end{tabular}

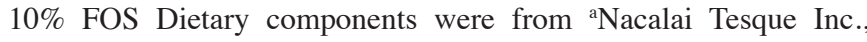
Kyoto, Japan, 'Sigma-Aldrich; Merck KGaA, Darmstadt, Germany, ${ }^{\mathrm{c}}$ Hiroshima Laboratory Animal Centre, Hiroshima, Japan and ${ }^{\mathrm{d}}$ Wako Pure Chemical Industries, Ltd., Osaka, Japan. The vitamin and mineral mixtures were used according to the American Institute for Nutrition formulation (AIN-93) (16). FOS, fructooligosaccharides.

was increased from 20 to $180^{\circ} \mathrm{C}$ at a rate of $20^{\circ} \mathrm{C} / \mathrm{min}$ and then maintained at $180^{\circ} \mathrm{C}$ for $4 \mathrm{~min}$. The flow rate of the carrier gas $\left(\mathrm{N}_{2}\right)$ was $1.0 \mathrm{ml} / \mathrm{min}$ (split ratio 10:1). Standard curves were linear across four different concentrations $(0.00,3.25,6.50$ and $13.00 \mathrm{mmol} / \mathrm{l})$ of ethanol $\left(\mathrm{R}^{2}>0.99\right)$, and used for the assay of ethanol levels in the samples.

Cecum microflora. For the analysis of intestinal microflora, bacterial genomic DNA was isolated from the cecum digesta using an UltraClean ${ }^{\mathrm{TM}}$ Fecal DNA extraction kit (MO BIO Laboratories; Qiagen, Inc., Valencia, CA, USA) according to the manufacturer's instructions. The purified DNA was eluted in $100 \mu$ l elution buffer ( $5 \mathrm{mM}$ Tris- $\mathrm{HCl}, \mathrm{pH} 8.5)$, and the quality and quantity of DNA were determined by measuring the absorbance at 260 and $280 \mathrm{~nm}$ using a NanoDrop ND-1000 spectrophotometer (NanoDrop Technologies; Thermo Fisher Scientific, Inc., Wilmington, DE, USA). Samples were stored at $-20^{\circ} \mathrm{C}$. Real-time quantitative polymerase chain reaction (qPCR) was performed to investigate the variation in the total number of bacteria, including of Bifidobacterium spp. and Lactobacillus spp. The $16 \mathrm{~S}$ rRNA primers for Bifidobacterium spp. and Lactobacillus spp. used for qPCR have been described previously (14). The total volume of reagent mixture for each PCR was $20 \mu 1$ [ $4.4 \mu \mathrm{l}$ distilled water, $10 \mu \mathrm{l}$ Master mix (Takara Bio, Inc., Otsu, Japan), $2 \mu 1$ Plus solution (Takara Bio, Inc., Otsu, Japan), $0.8 \mu \mathrm{l}$ of each forward and reverse primer and $2 \mu 1$ DNA]. The reaction conditions were as follows: $95^{\circ} \mathrm{C}$ for $30 \mathrm{sec}$, followed by 40 cycles at $95^{\circ} \mathrm{C}$ for $5 \mathrm{sec}, 55^{\circ} \mathrm{C}$ for $15 \mathrm{sec}$ and $72^{\circ} \mathrm{C}$ for $30 \mathrm{sec}$. The fluorescent products were detected at the last step of each cycle. Melting curve analysis was performed following amplification to distinguish the targeted PCR product from the non-targeted PCR products. Data were analysed using the second derivative maximum method of the StepOneTM Real-time PCR software version 2.3 (Applied Biosystems; Thermo Fisher Scientific, Inc.). The copy 

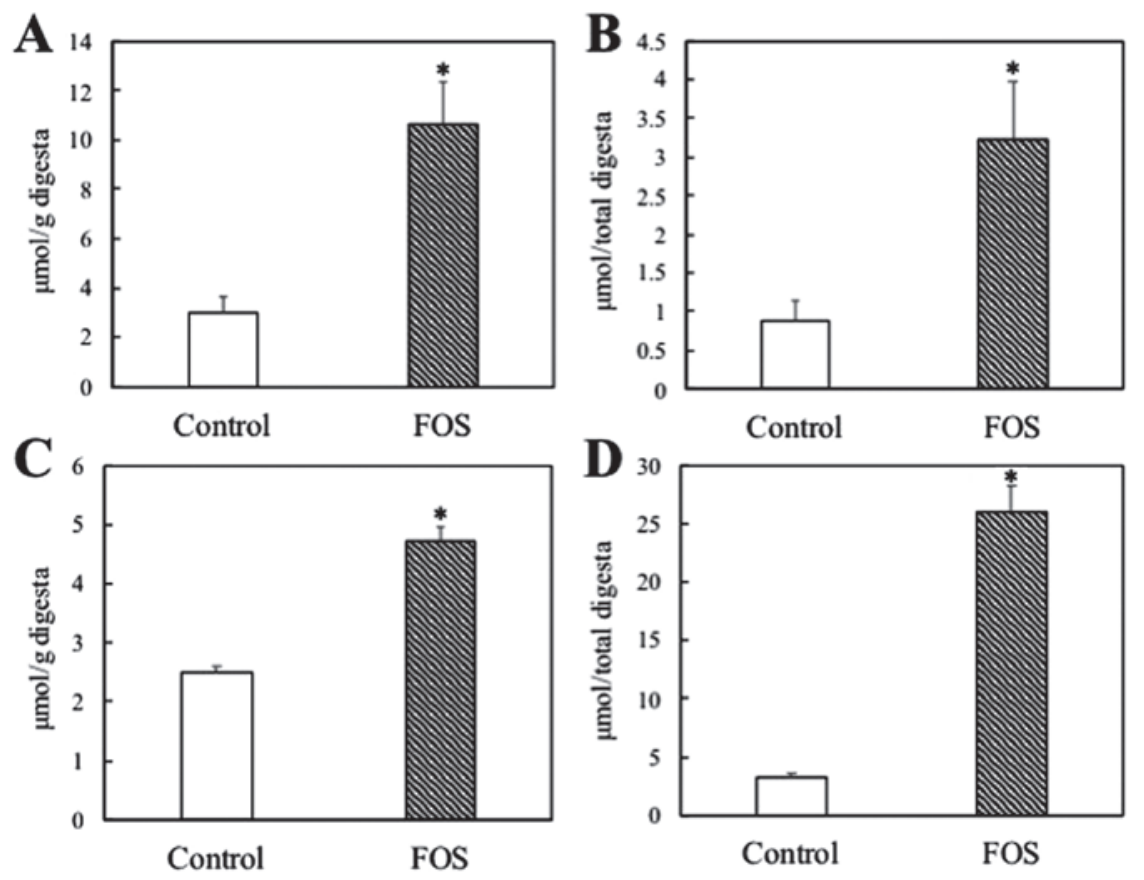

Figure 1. Effect of dietary FOS on ethanol levels in the ileum and cecum digesta of rats. (A) Ethanol levels per gram of ileum digesta; (B) ethanol levels in total ileum digesta; (C) ethanol levels per gram of cecum digesta; (D) ethanol levels in total cecum digesta. Values represent the means \pm standard error of the mean $(\mathrm{n}=8) .{ }^{*} \mathrm{P}<0.01$ vs. control group by Student's t-test. FOS, fructooligosaccharides.

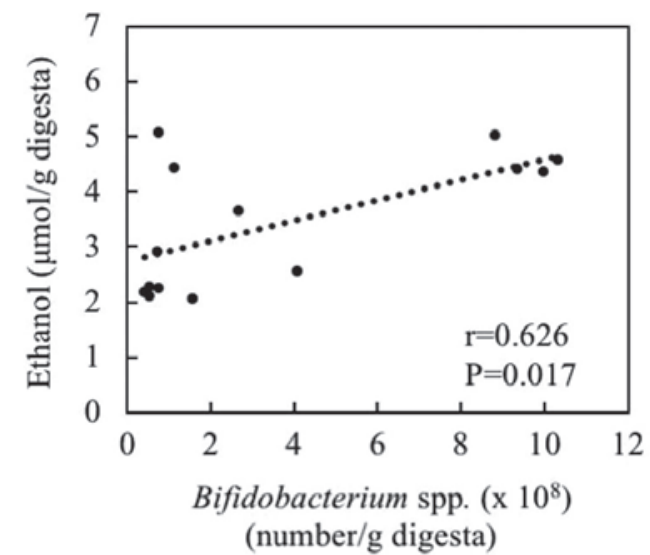

Figure 2. Association of ethanol levels with number of Bifidobacterium spp. per gram of cecum digesta.

numbers of Bifidobacterium spp. and Lactobacillus spp.//1 were determined for the standard plasmid solution of these bacteria [(cut standard plasmid mixture $\mathrm{ng} / \mu \mathrm{l}) \mathrm{x}$ (molecules $\left.\mathrm{bp} / 1.0 \times 10^{9} \mathrm{ng}\right) \times(1 / 660 \mathrm{DNA}$ length $\mathrm{bp} /$ plasmid $)=$ plasmid copies/ $\mu 1]$ (14). Real-time qPCR products were run as five 10 -fold serial dilutions of each standard mixture to compare the threshold cycle number with the copy number of the target sequence and to generate standard curves for the quantification of unknown samples. Typically, standard curves were linear across five orders of magnitude $\left(\mathrm{R}^{2}>0.98\right)$.

Cecum SCFAs. Cecum SCFAs were measured according to an internal standard method using high-performance liquid chromatography (HPLC; L-2130; Hitachi, Ltd., Tokyo, Japan) equipped with an Aminex HPX-87H ion exclusion column
(7.8 mm internal diameter x $30 \mathrm{~cm}$; Bio-Rad, Laboratories, Hercules, CA, USA) (18). Briefly, $500 \mathrm{mg}$ cecum digesta was homogenised in $5 \mathrm{ml} 50 \mathrm{mmol} / 1 \mathrm{H}_{2} \mathrm{SO}_{4}$ containing $10 \mathrm{mmol} / \mathrm{l}$ 2,2-dimethyl butyric acid as an internal standard and subsequently centrifuged at $17,000 \mathrm{x} \mathrm{g}$ at $2^{\circ} \mathrm{C}$ for $20 \mathrm{~min}$. The supernatant was ultrafiltered, and the filtrate was applied to the HPLC column.

Statistical analysis. Data are expressed as the mean \pm standard error of the mean. Data were analyzed by Student's t-test after statistical outliers were removed using the Smirnov-Grubbs rejection test. In addition, association of cecum ethanol levels with cecum Bifidobacterium and SCFA levels was assessed using Spearman rank correlation analysis. The data analysis was performed using Excel Statistics 2010 for Windows (Microsoft Corporation, Redmond, WA, USA). For all tests, $\mathrm{P}<0.05$ was considered to indicate statistical significance.

\section{Results}

Food intake and body weight. Dietary manipulation had no significant effect on total food intake over 14 days or on final body weight $(\mathrm{P}>0.05$; Table II).

Intestinal and serum ethanol levels. The weights of the ileum digesta were unaffected by FOS intake (control vs. FOS: $0.30 \pm 0.02$ vs. $0.26 \pm 0.05 \mathrm{~g} ; \mathrm{P}>0.05$ ). Ethanol levels per gram of ileum digesta were significantly increased 3.5-fold by FOS intake $(\mathrm{P}<0.01$; Fig. 1A). In the total ileum digesta, FOS intake significantly increased ethanol levels by 3.6 -fold $(\mathrm{P}<0.01$; Fig. 1B). The weights of the cecum digesta were markedly increased 4.2-fold by FOS intake (control vs. FOS: $1.33 \pm 0.06$ vs. $5.54 \pm 0.48 \mathrm{~g} ; \mathrm{P}<0.01)$. The ethanol levels per gram of cecum 
Table II. Effects of dietary FOS on the copy numbers of beneficial bacteria and levels of SCFAs in the cecum digesta of rats.

\begin{tabular}{lcc} 
& \multicolumn{2}{c}{ Dietary group } \\
\cline { 2 - 3 } Variable & Control & $10 \%$ FOS \\
\hline Final body weight, g & $198.1 \pm 1.9$ & $193.9 \pm 3.2$ \\
Total food intake over 14 days, g & $225.0 \pm 0.0$ & $225.0 \pm 0.0$ \\
Bacteria (numbers/g digesta) & & \\
Bifidobacterium spp. $\left(x 10^{8}\right)$ & $1.4 \pm 0.5$ & $5.9 \pm 1.8^{\mathrm{a}^{*}}$ \\
Lactobacillus spp. $\left(x 10^{9}\right)$ & $11.5 \pm 3.1$ & $7.5 \pm 4.7$ \\
Bacteria (numbers/total digesta) & & \\
Bifidobacterium spp. $\left(x 10^{8}\right)$ & $1.3 \pm 0.3$ & $31.6 \pm 9.6^{\mathrm{a}^{*}}$ \\
Lactobacillus spp. $\left(\mathrm{x} 10^{10}\right)$ & $1.5 \pm 0.3$ & $3.5 \pm 2.3$ \\
SCFAs $(\mu$ mol/g digesta) & & \\
Succinate & $36.2 \pm 7.3^{*}$ & $14.8 \pm 5.5^{\mathrm{a}^{* *}}$ \\
Lactate & $15.2 \pm 4.7^{*}$ & $35.8 \pm 4.6^{\mathrm{b}}$ \\
Acetate & $66.3 \pm 6.9$ & $24.4 \pm 6.2^{\mathrm{b}}$ \\
Propionate & $20.1 \pm 1.7$ & $33.7 \pm 4.9^{\mathrm{a}}$ \\
n-Butyrate & $15.5 \pm 1.8$ & $22.3 \pm 2.1^{\mathrm{a}}$ \\
Total SCFAs & $127.2 \pm 16.9^{* *}$ & $123.0 \pm 13.5^{* *}$ \\
SCFAs ( $\mu$ mol/total digesta) & & \\
Succinate & $49.0 \pm 11.2^{*}$ & $73.1 \pm 23.9^{* *}$ \\
Lactate & $19.3 \pm 4.7^{*}$ & $195.2 \pm 29.4^{\mathrm{b}}$ \\
Acetate & $86.0 \pm 7.5$ & $119.7 \pm 23.7$ \\
Propionate & $26.3 \pm 1.9$ & $189.7 \pm 34.6^{\mathrm{b}}$ \\
n-Butyrate & $20.3 \pm 2.1$ & $119.7 \pm 11.5^{\mathrm{b}}$ \\
Total SCFAs & $164.7 \pm 19.4^{* *}$ & $656.2 \pm 66.5^{\mathrm{b}^{* *}}$ \\
\hline & & \\
\hline & &
\end{tabular}

Values represent the means \pm standard error of the mean $(n=6-8)$. and ${ }^{* *}$ indicate studies using 6 and 7 animals, respectively, and others are the data of 8 animals; outlier data were omitted from certain studies following confirmation with the Smirnov-Grubbs rejection test. ${ }^{\mathrm{a}} \mathrm{P}<0.05$ and ${ }^{\mathrm{b}} \mathrm{P}<0.01$ vs. control determined by Student's t-test. FOS, fructooligosaccharides; SCFAs, short-chain fatty acids.

digesta were significantly increased 1.9 -fold by FOS intake ( $\mathrm{P}<0.01$; Fig. 1C). In the total cecum digesta, ethanol levels were markedly increased by FOS intake by 7.9 -fold $(\mathrm{P}<0.01$; Fig. 1D). Serum ethanol levels were marginally but significantly increased by FOS intake (control vs. FOS: $2.63 \pm 0.05$ vs. $2.84 \pm 0.07 \mathrm{mM}$; $\mathrm{P}<0.05)$.

Cecum bacteria and SCFAs. Compared with the control group, the copy numbers of Bifidobacterium per gram of cecum digesta were markedly increased 4.2-fold in the FOS group $(\mathrm{P}<0.05$; Table II). However, the numbers of Lactobacillus per gram of cecum digesta were unaffected $(\mathrm{P}>0.05)$. The numbers of Bifidobacterium were positively correlated with ethanol level per gram of cecum digesta $(\mathrm{r}=0.626, \mathrm{P}=0.017$; Fig. 2).

Succinate and acetate levels per gram of cecum digesta were decreased by FOS intake (2.5- and 2.7-fold decrease, respectively; $\mathrm{P}<0.05$; Table II). By contrast, FOS intake significantly increased lactate, propionate and n-butyrate levels per gram of cecum digesta (2.4-, 1.7- and 1.4-fold, respectively; $\mathrm{P}<0.05$; Table II). In the total cecum digesta, the levels of lactate, propionate, n-butyrate and total SCFAs were markedly increased by the FOS diet (10.3-, 7.3-, 6.0- and 4.0-fold, respectively, $\mathrm{P}<0.01)$. The ethanol levels per gram of cecum digesta were negatively correlated with acetate levels $(r=-0.558$, $\mathrm{P}=0.025$; Fig. $3 \mathrm{~A}$ ) and positively correlated with lactate levels $(r=0.691, P=0.006$; Fig. 3B). However, ethanol levels were not significantly correlated with the level of propionate $(\mathrm{r}=0.449$, $\mathrm{P}=0.081$; Fig. 3 C) or butyrate ( $\mathrm{r}=0.493, \mathrm{P}=0.052$; Fig. 3D).

\section{Discussion}

The present study demonstrated, to the best of our knowledge for the first time, that FOS intake significantly increased ethanol levels in the intestinal digesta of rats without direct consumption of ethanol. The results further indicated that the cecum levels of ethanol were associated with Bifidobacterium numbers. Previous in vitro studies have indicated that ethanol is produced by Bifidobacterium from glucose or FOS $(2,3)$. Other in vitro studies have suggested that the addition of FOS stimulates the growth of Bifidobacterium $(6,7)$. Therefore, the increase in intestinal ethanol levels may, at least in part, be mediated by an increased abundance of Bifidobacterium bacteria in the intestines of rats fed FOS. However, a previous study suggested ethanol is degraded to acetate by intestinal bacteria such as Bifidobacterium and Lactobacillus (19). Taken together, it may be hypothesized that Bididobacterium serves a role in the degradation of ethanol in rats following consumption of ethanol, while Bifidobacterium may stimulate the production of ethanol in rats lacking consumption of ethanol. Since the abundance of cecum Lactobacillus was unaffected by FOS in the current study, the possibility of the involvement of Lactobacillus in the mechanisms underlying the increase in intestinal ethanol was negated. At present, the possibilities of higher production and/or lower degradation of ethanol by other intestinal bacteria in rats fed FOS remains to be examined. A recent metagenome study demonstrated that FOS intake increased or decreased the abundance of a variety of bacteria in addition to Bifidobacterium in rats (20). Accordingly, further in vitro study is necessary to investigate if each of the intestinal bacteria present can produce or degrade ethanol.

Bifidobacterium is established to be a producer of lactate and ethanol $(2,3)$. The current study also identified a significant positive correlation between the cecum levels of ethanol and lactate. It has been suggested that lactate is produced from pyruvate and that ethanol is produced from acetyl-CoA, a metabolite of pyruvate, in Bifidobacterium $(2,3)$. Therefore, higher production of lactate may be associated with a higher production of ethanol. Further studies should be conducted to elucidate the underlying mechanisms of higher production of ethanol following elevated FOS intake.

High ethanol intake is well established to cause toxic effects and increase the risk of several diseases, including liver disease, coronary heart diseases and cancer (21). However, previous studies have suggested that low or moderate ethanol intake is associated with a lower risk of developing diseases including coronary heart disease, brain diseases and cancers $(10,11)$. Previous experimental 

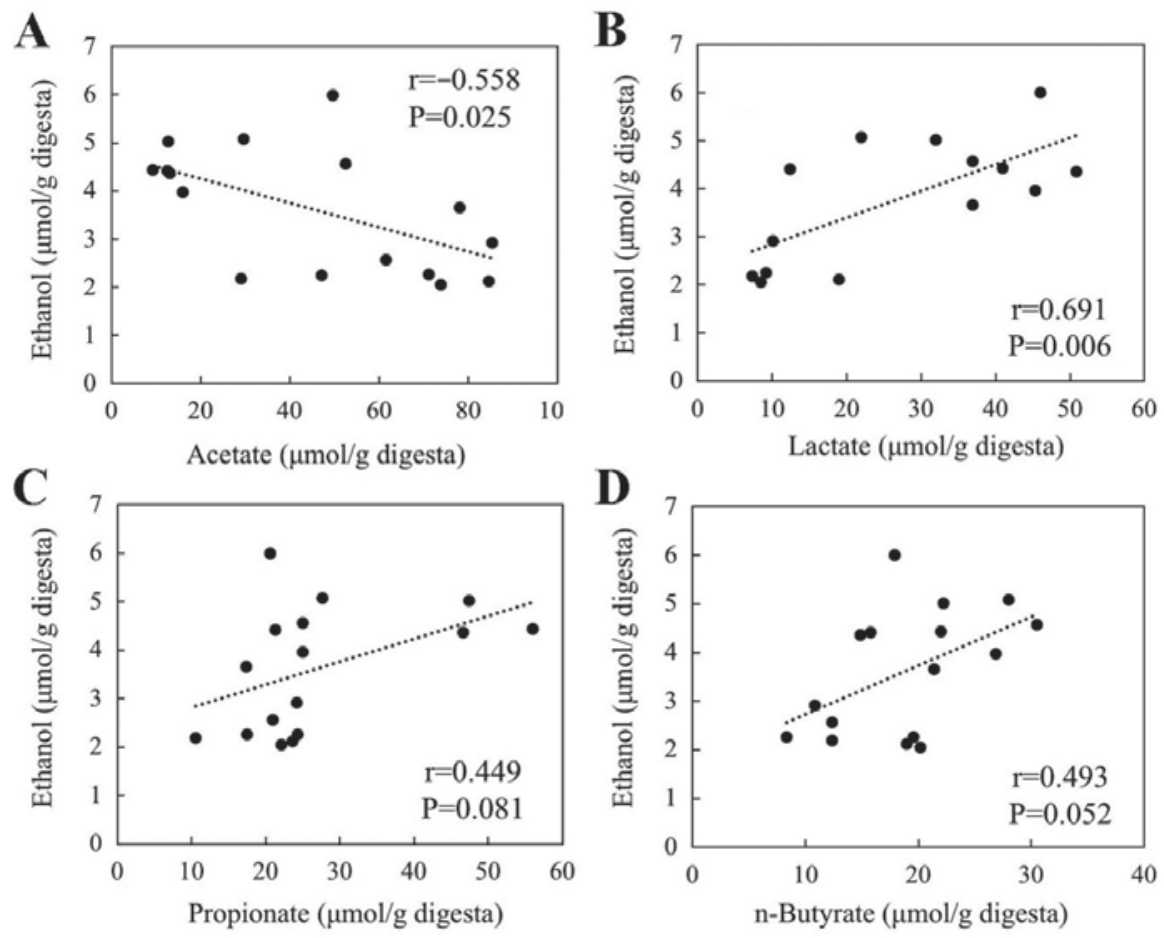

Figure 3. Association of ethanol levels with the levels of (A) acetate, (B) lactate, (C) propionate and (D) n-butyrate per gram of cecum digesta.

studies by our group have also suggested beneficial effects of a low ethanol dose on liver function and aging in rodents $(22,23)$. Therefore, further studies are necessary to investigate whether increased ethanol levels in rats fed FOS are equivalent to the toxic or beneficial levels reported in animals, and to determine the overall ethanol production rate by intestinal microflora in rats fed FOS. In this regard, a study by Zhong et al (24) reported that rats administered a Lieber-DeCarli liquid diet mixed with $5 \%$ (w/v) ethanol for 8 weeks exhibited an elevation in cecum ethanol levels from 2-3 to $20-30 \mu \mathrm{mol} / \mathrm{g}$ digesta. The increased intestinal ethanol levels were associated with fatty liver disease pathology and reduced intestinal barrier function (24). Their results imply that increased levels of cecum ethanol (>20 $\mu$ mol per gram of digesta) in rats fed ethanol may be harmful for the host. A more recent study using Caco- 2 cells monolayers as an in vitro tight junction model indicated that the addition of $6 \%(\mathrm{v} / \mathrm{v}, 1 \mathrm{mmol} / \mathrm{ml})$ ethanol to the culture medium decreased intestinal cell barrier function, although cell viability and lactate dehydrogenase release (cytotoxicity) were unaffected (25). The current study identified the production of 4-5 $\mu \mathrm{mol}$ ethanol per gram of cecum digesta in rats fed FOS. Thus, the levels of ethanol in FOS-fed rats appear to be lower than the harmful levels of ethanol reported $(24,25)$. Further study is necessary to investigate whether the increased ethanol levels in the cecum of rats fed FOS are close to the beneficial levels in those administered low-dose ethanol in our previous studies $(22,23)$.

In conclusion, the current study demonstrated that dietary FOS increased ethanol levels in the intestinal digesta of rats fed a HF diet. The results imply that FOS intake has an impact on intestinal health by increasing ethanol levels. The findings also indicate the importance of dietary prebiotics as an envi- ronmental factor in alcohol-use disorders and may provide novel insight into the link between prebiotics and diseases related to alcohol consumption.

\section{Acknowledgements}

This study was presented as part of an abstract at the 17th Global Dieticians and Nutritionists Annual Meeting October 32017 in Kuala Lumpur, Malaysia and published under Masahiro Yamaguchi et al in J Nutr Food Sci 7: 6, 2017.

\section{Funding}

The current study was financially supported by the HIRAKU consortium, Hiroshima University.

\section{Availability of data and materials}

The analyzed data sets generated during the study are available from the correspondence author on reasonable request.

\section{Authors' contributions}

MY, YY and NK contributed to the study design, MY, YY, MA and YO acquired the data. MY and TK analyzed and interpreted the data. MY and NK wrote the manuscript and approved the contents of the manuscript. The final version of the manuscript has been read and approved by all authors.

\section{Ethics approval and consent to participate}

The study protocol was approved by the institutional ethics committee of Hiroshima University (approval no: C15-12). 


\section{Consent for publication}

Not applicable.

\section{Competing interests}

The authors declare that they have no competing interests.

\section{References}

1. Cope K, Risby T and Diehl AM: Increased gastrointestinal ethanol production in obese mice: Implications for fatty liver disease pathogenesis. Gastroenterology 119: 1340-1347, 2000.

2. Van der Meulen R, Adriany T, Verbrugghe K and De Vuyst L: Kinetic analysis of bifidobacterial metabolism reveals a minor role for succinic acid in the regeneration of NAD+ through its growth-associated production. Appl Environ Microbiol 72: 5204-5210, 2006.

3. Rivière A, Selak M, Lantin D, Leroy F and De Vuyst L: Bifidobacteria and butyrate producing colon bacteria: Importance and strategies for their stimulation in the human gut. Front Microbiol 7: 979-990, 2016.

4. Elshaghabee FM, Bockelmann W, Meske D, de Vrese M, Walte HG, Schrezenmeir J and Heller KJ: Ethanol production by selected intestinal microorganisms and lactic acid bacteria growing under different nutritional conditions. Front Microbiol 7: 47-59, 2016

5. Barona M, Roy D and Vuillemard JC: Biochemical characteristics of fermented milk produced by mixed cultures of lactic starters and bifidobacteria. Lait 80: 465-478, 2000.

6. Sharp R, Fishbain S and Macfarlane GT: Effect of short-chain carbohydrates on human intestinal bifidobacteria and Escherichia coli in vitro. J Med Microbiol 50: 152-160, 2001

7. Mao B, Li D, Zhao J, Liu X, Gu Z, Chen YQ, Zhang H and Chen W: In vitro fermentation of fructooligosaccharides with human gut bacteria. Food Funct 6: 947-954, 2015.

8. Cummings JH, Macfarlane GT and Englyst HN: Prebiotic digestion and fermentation. Am J Clin Nutr 73: 415S-420S, 2001

9. Pandey KR, Naik SR and Vakil BV: Probiotics, prebiotics and synbiotics- a review. J Food Sci Technol 52: 7577-7587, 2015.

10. Fekjaer HO: Alcohol - a universal preventive agent? A critical analysis. Addiction 108: 2051-2057, 2013.

11. Muñoz G, Urrutia JC, Burgos CF, Silva V, Aguilar F, Sama M, Yeh HH, Opazo C and Aguayo LG: Low concentrations of ethanol protect against synaptotoxicity induced by $A \beta$ in hippocampal neurons. Neurobiol Aging 36: 845-856, 2015.

12. Hamilton MK, Ronveaux CC, Rust BM, Newman JW, Hawley M, Barile D, Mills DA and Raybould HE: Prebiotic milk oligosaccharides prevent development of obese phenotype, impairment of gut permeability, and microbial dysbiosis in high fat-fed mice. Am J Physiol Gastrointest Liver Physiol 312: G474-G487, 2017.

13. Tunapong W, Apaijai N, Yasom S, Tanajak P, Wanchai K, Chunchai T, Kerdphoo S, Eaimworawuthikul S, Thiennimitr P, Pongchaidecha A, et al: Chronic treatment with prebiotics, probiotics and synbiotics attenuated cardiac dysfunction by improving cardiac mitochondrial dysfunction in male obese insulin resistant rats. Eur J Nutr 57: 2091-2104, 2018.
14. Yang Y, Iwamoto A, Kumrungsee T, Okazaki Y, Kuroda M, Yamaguchi S and Kato N: Consumption of an acid protease derived from Aspergillus oryzae causes bifidogenic effect in rats. Nutr Res 44: 60-66, 2017.

15. Coudray C, Tressol JC, Gueux E and Rayssiguier Y: Effects of inulin-type fructans of different chain length and type of branching on intestinal absorption and balance of calcium and magnesium in rats. Eur J Nutr 42: 91-98, 2003.

16. Reeves PG, Nielsen FH and Fahey GC Jr: AIN-93 purified diets for laboratory rodents: Final report of the American Institute of Nutrition ad hoc writing committee on the reformulation of the AIN-76A rodent diet. J Nutr 123: 1939-1951, 1993.

17. Heit C, Eriksson P, Thompson DC, Charkoftaki G, Fritz KS and Vasiliou V: Quantification of Neural Ethanol and Acetaldehyde Using Headspace GC-MS. Alcohol Clin Exp Res 40: 1825-1831, 2016.

18. Okazaki Y, Tomotake H, Tsujimoto K, Sasaki M and Kato N: Consumption of a resistant protein, sericin, elevates fecal immunoglobulin A, mucins, and cecal organic acids in rats fed a high-fat diet. J Nutr 141: 1975-1981, 2011.

19. Nosova T, Jousimies-Somer H, Jokelainen K, Heine R and Salaspuro M: Acetaldehyde production and metabolism by human indigenous and probiotic Lactobacillus and Bifidobacterium strains. Alcohol Alcohol 35: 561-568, 2000.

20. Liu J, Kandasamy S, Zhang J, Kirby CW, Karakach T, Hafting J, Critchley AT, Evans F, Prithiviraj B and Prithiviraj B: Prebiotic effects of diet supplemented with the cultivated red seaweed Chondrus crispus or with fructo-oligo-saccharide on host immunity, colonic microbiota and gut microbial metabolites. BMC Complement Altern Med 15: 279, 2015

21. Rehm J, Gmel GE Sr, Gmel G, Hasan OSM, Imtiaz S, Popova S, Probst C, Roerecke M, Room R, Samokhvalov AV, et al: The relationship between different dimensions of alcohol use and the burden of disease-an update. Addiction 112: 968-1001, 2017.

22. Osaki A, Okazaki Y, Kimoto A, Izu H and Kato N: Beneficial effect of a low dose of ethanol on liver function and serum urate in rats fed a high-fat diet. J Nutr Sci Vitaminol (Tokyo) 60: 408-412, 2014

23. Kimoto A, Izu H, Fu C, Suidasari S and Kato N: Effects of low dose of ethanol on the senescence score, brain function and gene expression in senescence-accelerated mice 8 (SAMP8). Exp Ther Med 14: 1433-1440, 2017.

24. Zhong W, Li Q, Zhang W, Sun Q, Sun X and Zhou Z: Modulation of Intestinal Barrier and Bacterial Endotoxin Production Contributes to the Beneficial Effect of Nicotinic Acid on Alcohol-Induced Endotoxemia and Hepatic Inflammation in Rats. Biomolecules 5: 2643-2658, 2015.

25. Chopyk DM, Kumar P, Raeman R, Liu Y, Smith T and Anania FA: Dysregulation of junctional adhesion molecule-A contributes to ethanol-induced barrier disruption in intestinal epithelial cell monolayers. Physiol Rep 5: e13541, 2017. 